\title{
Correspondence
}

\section{Neonatal urological ultrasound}

Sir,

It is a cause for concern that I find the statement 'all patients underwent renography and cystography, with anatomical localisation by intravenous urography and sometimes antegrade pyelography', in a paper published in 1989. ${ }^{1}$ The obsessive desire for full assessment should not override management based on a primary division of patients into those requiring early intervention and those requiring careful follow up. For example, dilatation of one renal tract in a neonate may resolve spontaneously without surgery. There is no single imaging pathway that is correct for all neonates with urinary abnormalities. Prudence in the use of ionising radiation dictates the need for individual assessment by surgeon and radiologist followed by the use of investigations relevant to the individual problem.

\section{R K LeVICK \\ Paediatric Radiology Department, Sheffield Children's Hospital, Western Bank, Sheffield S10 2 TH}

\author{
Reference \\ ${ }^{1}$ Clarke NW, Gough DCS, Cohen SJ. Neonatal urological \\ ultrasound: diagnostic inaccuracies and pitfalls. Arch Dis Child \\ 1989;64:578-80.
}

\section{Dr Clarke comments:}

While we agree that not all cases with urinary tract dilatation at birth require early surgical intervention, we maintain that in order to be sure which cases are best managed conservatively, accurate diagnosis must first be made.

Regarding the example quoted by Dr Levick, we would certainly not advocate the full investigation of a neonate with a transiently dilated urinary tract. If the dilatation persists, however, the information provided by an ultrasound scan is insufficient to provide accurate diagnosis and plan appropriate management. This fact is confirmed by the results of our paper.

We do tailor our investigations to the individual child but it is inappropriate to dispense with definite imaging as this is the only way to make specific diagnosis. To settle for anything less is to adopt a policy of 'best guess'. This is neither appropriate nor safe in current urological practice.

Sir,

In a recent paper by Clarke et al investigating neonatal urological scanning it was pointed out that this is not absolutely reliable and that a number of misdiagnoses might lead to major morbidity. ${ }^{1}$ We report a case that emphasises the message.
A girl was born at full term after the antenatal scan had shown a multicystic right kidney and a normal left kidney. Postnatal scan and intravenous urography showed dilatation of the collecting system. She was referred to a regional paediatric hospital. There, ultrasound showed a right duplex system with dilatation and a large ureterocele and a dimercaptosuccinic acid (DMSA) isotope scan confirmed this finding with little function in the upper pole.

She was listed for a right upper heminephrouretectomy three months later. At operation she was found to have a Wilms' tumour occupying the right upper calices and she underwent nephrectomy. The tumour was of favourable histology and as there was no other spread she received weekly courses of vincristine only for 10 weeks.

This case confirms the view that full investigation of all antenatally detected urinary tract abnormalities must be undertaken to prevent missing cases with a potentially lethal outcome.

\section{Reference \\ ${ }^{1}$ Clarke NW, Gough DCS, Cohen SJ. Neonatal urological ultrasound: diagnostic inaccuracies and pitfalls. Arch Dis Child 1989;64:578-80.}

A N CAMPBell and J R OWEN Royal Preston Hospital, Sharoe Green Lane, Preston PR2 4HT

Sir,

Clarke et al are quite correct to emphasise that ultrasound alone is rarely enough to initiate treatment of renal tract abnormalities detected antenatally. ${ }^{1}$ Their paper, however, does not define the precise role of ultrasound and underestimates its diagnostic capabilities in experienced hands.

The role of antenatal ultrasound is twofold. Firstly to detect abnormalities incompatible with a normal life expectancy so that termination of pregnancy can be offered. A recent study has shown good specificity of ultrasound in this situation. ${ }^{2}$ Secondly to forewarn of structural renal abnormalities so that these can be investigated after birth. The specific cause for renal tract dilatation at this stage is not important.

Postnatally the situation is quite different. Ultrasound clearly shows pelvicaliceal and ureteric dilatation, bladder volume and wall thickness, the presence of diverticula and ureteroceles, and dilatation of the posterior urethra in cases of posterior urethral valves. Dilatation of collecting systems does not necessarily infer obstruction and further imaging is necessary to exclude reflux or non-obstructed dilated systems. Pelviureteric junction obstruction often has a characteristic appearance on ultrasound, however, and it is surprising that Clarke et al found this abnormality to be accurately diagnosed in only 14 of 35 cases. We are 